Ann. Biol. anim. Bioch. Biophys., I974, 14 (4-A), 72I-727.

NOTE

\title{
INFLUENCE DE TEMPÉRATURES ET D'HYGROMÉTRIES ÉLEVÉES PENDANT UNE PARTIE DE LA JOURNÉE SUR LA THERMORÉGULATION DU VEAU PRÉRUMINANT A L'ENGRAIS ( $\left.{ }^{1}\right)$
}

\author{
J. C. BOUVIER, J. ESPINOSA-MOLINER $\left({ }^{2}\right)$ et M. VERMOREL \\ avec la collaboration technique de G. Moins et R. Souchet \\ Laboratoire d' Ĺtude du Métabolisme énergétique, \\ Centre de Recherches de Clermont-Ferrand, I. N.R. A., \\ Theix, 63110 Beaumont
}

\section{RÉSUMÉ}

Treize veaux mâles de racc Frisonne ont été exposés à des températures de 28 ou $33^{\circ} \mathrm{C}$ et des humidités relatives de 60 à $90 \mathrm{p}$. I oo pendant 7 heures puis à une température de $19^{\circ} \mathrm{C}$ et $75 \mathrm{p}$. I 100 HR pendant le reste de la journée. Les dépenses énergétiques des veaux ont été mesurées à l'aide de 2 chambres respiratoires du type circuit ouvert pendant 37 journées de traitement et 39 journées de référence.

Ce stress ne réduit pas l'appétit des veaux, provoque une augmentation de la dépense énergétique de $3 \mathrm{p}$. Ioo en moyenne pendant la période de stress et pendant la journée entière (tabl, I). L'influence du stress se prolonge pendant une durée variable, de 2 à 8 heures suivant les animaux (fig. I).

La quantité d'eau totale évaporéc par la voie cutanée et la voie respiratoire est augmentée de $33 \mathrm{p}$. Ioo pendant la journée entjère et doit être doublée pendant la période de stress (tabl. I). La chaleur latente est augmentée par le stress : 54,5 contre $42,5 \mathrm{p}$. Ioo pendant la journée entière et 78 contre $42,5 \mathrm{p}$. Ioo pendant la période de stress.

La fréquence respiratoire est accrue par l'augmentation de la température ambiante et davantage encore par l'humidité relative de l'air (tabl. 2). Pour une température donnée, l'augmentation est plus importante et plus rapide lorsque l'hygrométrie est élevée. Ce phénomène accompagne une augmentation plus importante de la température rectale (fig. 2).

(1) Co travail a fait l'objet d'une partie de la Thèse de Doctorat de Spécialité de J. Espinosa-Moliner. (2) Adresse actuelle : Centro Nacional de Investigaciones Cientificas, Cubanacan, La Habana (Cuba). 


\section{I. - INTRODUCTION}

Les conditions climatiques correspondant à des températures et des hygrométries élevées constituent souvent un facteur limitant des performances des animaux en production intensive, même dans les régions tempérées. En dehors des travaux de l'équipe du Missouri sur les jeunes bovins ruminants (KIBLER, I957; KIBLER, I962; JoHNSTON et al., I963) la plupart des études de bioclimatologie concernant le veau préruminant ou ruminant ont été réalisées dans des conditions

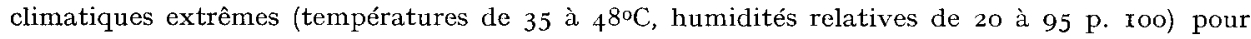
accentuer les réponses physiologiques des animaux (FERGUson et Dowling, I955; KLemm et Robinson, I955; McLean, I963 ; Branca et Hales, 1970). Cependant ces conditions expérimentales sont très éloignées de celles rencontrées habituellement et ne tiennent compte ni de l'évolution des facteurs climatiques au cours de la journée ni des variations nycthémérales de la production de chaleur des animaux. Ainsi VAN Es (1967) a constaté que des vaches laitières et des veaux à l'engrais peuvent supporter des conditions climatiques défavorables $\left(30^{\circ} \mathrm{C}\right)$ pendant une partie de la journée s'ils ont la possibilité de récupérer pendant le reste de la journée.

Compte tenu de ces observations nous avons étudié, chez le veau préruminant à l'engrais, l'influence d'un cycle de température et d'hygrométrie, se rapprochant de celui rencontré en été dans les étables d'engraissement, sur la thermogénèse, la thermoly'se, la température rectale et la fréquence respiratoire.

\section{II. - MATÉRIEI, ET MÉTHODES}

Les études ont été réalisées sur I 3 veaux mâles préruminants à l'engrais, de race Frisonne recevant un aliment d'allaitement classique selon un plan de rationnement intensif. L'aliment a été distribué au seau en 2 repas égaux, à 9 heures et à $\mathrm{I} 7$ heures, en quantité légèrement limitée pour éviter les refus et les fluctuations journalières de consommation. Les animaux, dont le poids était compris entre 80 et $155 \mathrm{~kg}$, ont été placés dans des chambres respiratoires du type circuit ouvert (VERMOREL et al., I973) et soumis aux conditions climatiques suivantes:

- $19^{\circ} \mathrm{C}$ et $75 \mathrm{p}$. Ioo d'humidité relative pendant 24 heures (journées de référence) ;

- 28 ou $32^{\circ} \mathrm{C}$ et 60 ou $90 \mathrm{p}$. Ioo d'humidité relative (HR) de $9 \mathrm{~h} 3^{\circ}$ à $\mathrm{I} 6 \mathrm{~h} 3^{\circ}$ et $\mathrm{I} 9^{\circ} \mathrm{C}$ et 75 p. roo HR pendant le reste de la journée (journées de traitement).

L'augmentation de la température demandait une demi-heure (de $9 \mathrm{~h}$ 3o à Io heures) mais l'humidité relative de $90 \mathrm{p}$. Ioo n'était obtenue qu'au bout de I h 30 ; de même, le retour aux conditions initiales demandait environ 45 minutes (de I6 h 30 à I 7 h I5). Les journées de traitement ont en général été encadrées par des journées de référence.

Les mesures des échanges respiratoires effectuées toutes les 2 minutes ont été cumulées sur des périodes d'une demi-heure. Le bilan hydrique du veau présente de larges variations journalières, même pour les journées de référence, en raison des différences de productions quotidiennes d'eau fécale (jusqu'à $500 \mathrm{~g}$ ) et d'urine ( $\mathrm{I}$ à $3 \mathrm{~kg}$ ). La production d'eau des veaux a donc été calculée à partir de la quantité d'eau condensée sur l'échangeur froid et de la variation d'hygrométrie de l'air au cours de son passage dans la chambre respiratoire (VERMOREL et al., 1973). Cette mesure n'a pas pu être effectuée sur la période correspondant au stress en raison de la condensation d'eau dans les gaines pour les hygrométries les plus élevées.

La température rectale a été déterminée à l'aide d'une thermistance et enregistrée toutes les 2 minutes. La fréquence respiratoire a été mesurée de visu à 3 reprises pendant une minute toutes les demi-heures.

\section{III. - RÉSULTATS E'T DISCUSSION}

$$
\text { I. - Thermogenèse }
$$

Les mesures des échanges respiratoires sont utilisables pour 39 journées de référence et 37 journées de traitement pendant lesquelles les veaux ont consommé complètement leur ration. Les conditions climatiques de ce traitement ne réduisent pas l'appétit des veaux mais provoquent en 
moyenne une augmentation significative $(\mathrm{P}<\mathrm{o,OI})$ de la consommation d'oxygène, de la production de gaz carbonique et de la production de chaleur, pendant la durée du stress (8 heures) et pendant la totalité de la journée (tabl. I).

\section{TABLEAU I}

Influence de températures et d'hygrométries élevées ( 28 à $33^{\circ} \mathrm{C}$, 60 à 90 p. Ioo $\mathrm{HR}$ ) sur les échanges respiratoires et la production de chaleur du veau préruminant sur la quantité d'eau évaporée el le pourcentage de chaleur dissipée sous forme latente

$$
\text { (moyenne } \pm \text { écart-type) }
$$

\begin{tabular}{|c|c|c|c|}
\hline \multirow{2}{*}{ Mesures } & \multirow{2}{*}{ Journée de référence } & \multicolumn{2}{|c|}{ Journée de traitement } \\
\hline & & Journée entière & $8 \mathrm{~h}$ de stress \\
\hline Production de $\mathrm{CO}_{2}$ (relatif) & 100 & $103,5 \pm 0,6$ & $105,1 \pm 0,7$ \\
\hline Consommation de $\mathrm{O}_{2}$ (relatif) & 100 & $102,7 \pm 0,6$ & $102,1 \pm 0,5$ \\
\hline Production de chaleur (relatif) & 100 & $102,9 \pm 0,6$ & $102,8 \pm 0,5$ \\
\hline Eau évaporée $\left(\mathrm{g} / \mathrm{m}^{2} / \mathrm{h}\right)$ & $70 \pm 6$ & $93 \pm 9$ & $11^{\prime} 0$ \\
\hline Chaleur latente p. 100 chaleur totale & $42,5 \pm 6,9$ & $54,5 \pm 6,9$ & 78 \\
\hline
\end{tabular}

Pour étudier l'influence des conditions climatiques appliquées pendant le traitement, en fonction de l'intensité du métabolisme, sur les échanges respiratoires des veaux, nous avons fait une analyse de covariance sur les consommations d'oxygène et les productions de gaz carbonique pendant les journées de référence et les journées de traitement pour les conditions suivantes :

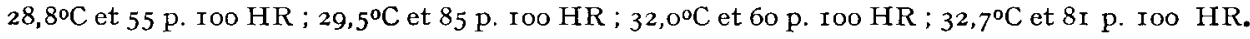
En raison peut-être du nombre réduit de données par traitement, il n'y a de différence significative ni entre les moyennes, ni entre les coefficients de régression (dans le cas de la production de gaz

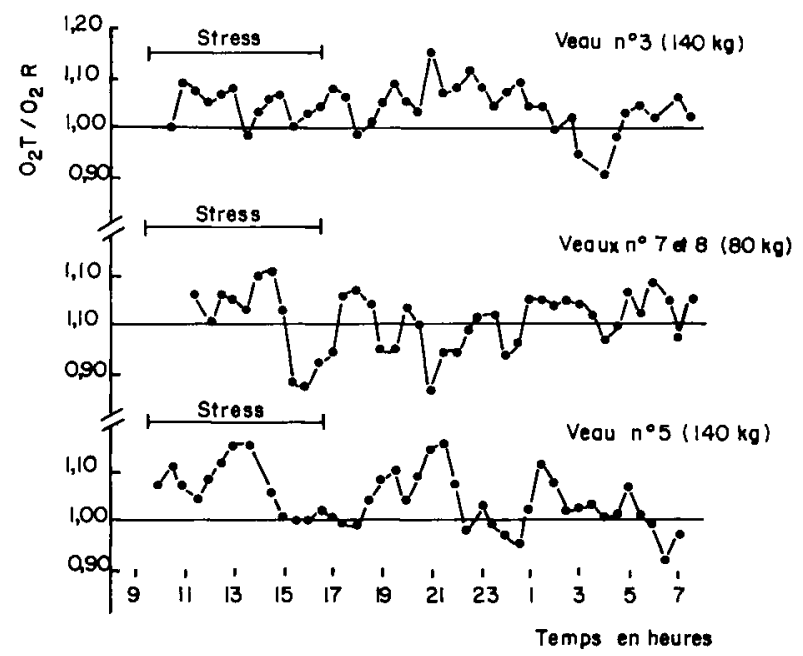

FIG. I. - Évolution du rapport entre les quantités d'oxygène consommé lors des journées de traitement $\left(\mathrm{O}_{2} \mathrm{~T}\right)\left(33^{\circ} \mathrm{C}, 80\right.$ p. Ioo d'humidité relative) et les journées de références $\left(\mathrm{O}_{2} \mathrm{R}\right)$ en fonction du temps

(Moyenne de 4 et 2 jours pour les veaux $n^{0} 3$ et 4 , moyenne d'une journée pour les veaux $n^{\circ} 7$ et 8 ) 
carbonique, respectivement I,034-0,994-1,020-I,I00). Ce dernier coefficient cependant semble indiquer que les animaux les plus lourds ou ceux qui ont le métabolisme énergétique le plus élevé sont plus sensibles que les autres aux conditions les plus défavorables $\left(32,7^{\circ} \mathrm{C}\right.$ et $8 \mathrm{I}$ p. roo $\left.\mathrm{HR}\right)$.

Le rapport des consommations d'oxygène, par période d'une demi-heure, pour les journées de traitement et les journées de références (fig. I) ne montre pas d'évolution nette au cours de la période de stress. L'augmentation moyenne de la production de chaleur ( $3 \pm 0,6 \mathrm{p}$. 1oo) est inférieure à l'accroissement de Io p. IOO observé par VAN Es (1967) au bout de 20 à 24 heures chez des veaux à l'engrais de $150 \mathrm{~kg}$ placés à des températures de $27^{\circ} \mathrm{C}$ et $95 \mathrm{p}$. Ioo $\mathrm{HR}$, ou $3^{\circ} \mathrm{C}$ et $75 \mathrm{p}$. roo $\mathrm{HR}$, lorsque la température rectale des animaux atteint $4^{\circ}{ }^{\circ} \mathrm{C}$.

Par ailleurs, l'influence du stress se prolonge pendant une durée de 5 à 8 heures chez les veaux $n^{0} 3$ et 5 pesant $\mathrm{I}_{4} \mathrm{o} \mathrm{kg}$; en revanche, les veaux $\mathrm{n}^{\circ} 7$ et 8 pesant $80 \mathrm{~kg}$ récupèrent plus rapidement et l'on peut considérer que 2 heures après la fin du stress la consommation d'oxygène est comparable à celle des journées de référence. Enfin, bien que l'augmentation de la production de chaleur soit la même pendant la durée du stress et pendant la totalité de la journée, nous n'avons pas observé d'effet de rémanence sur la journée de référence suivante.

\section{2. - Thermolyse}

La surface des veaux a été calculée à partir de leur poids à l'aide de la formule de Marthews et al. (1928) $\left(\mathrm{S}=0, \mathbf{I} 2 \mathrm{P}^{0,60}\right.$. Pour les journées de référence, la production d'eau par voie pulmonaire et par voie cutanée est en moyenne de ${ }_{1} 674 \pm I_{4} 2 \mathrm{~g} / \mathrm{m}^{2}$ et par jour. Cette valeur est très proche de celle que l'on peut calculer ( $1760 \mathrm{~g}$ ) à partir des données de VAN Es (1967) pour un veau de $130 \mathrm{~kg}$ placé à une température de $20^{\circ} \mathrm{C}$

Le stress climatique provoque une augmentation significative $(\mathrm{P}<$ o, ooI $)$ de 33 p. roo en moyenne de la quantité d'eau produite pendant la journée entière (tabl. I). Si l'on admet, au vu de l'évolution de la température rectale et de la fréquence respiratoire, qu'après le stress d'une durée de 8 heures la production d'eau est comparable à celle de la journée de référence, on peut calculer que pendant la durée du stress la production d'eau par voie pulmonaire et par voie cutanée est doublée ( $\mathrm{r} 4 \mathrm{O} \mathrm{g}$ contre $70 \mathrm{~g} / \mathrm{m}^{2}$ et par heure). Cette valeur est en accord avec celles que l'on peut calculer (I 47 à $\mathrm{I} 60 \mathrm{~g} / \mathrm{m}^{2}$ et par heure) à partir des données de McLean (1963) et de Bianca et Hales (1970) correspondant à des bovins d'un an placés à dzs températures de 30 à $33^{\circ} \mathrm{C}$.

La quantité de chaleur dissipée sous forme latente a été calculée en estimant à $0,6 \mathrm{kcal} / \mathrm{g}$ la chaleur latente de vaporisation de l'eau. Le stress climatique d'une durée de 8 heures entraîne une augmentation significative $(\mathbf{P}<0,0 \mathrm{I})$ des pertes de chaleur sous forme latente pendant la totalité de la journée $(54,5$ contre $42,5 \mathrm{p}$. Ioo) et pendant la période de stress ( 78 contre $42,5 \mathrm{p}$. IOO) selon l'hypothèse précédente. En fait, si l'on considère que pendant le stress climatique une partie de la chaleur n'est pas évacuée et provoque une élévation de la température corporelle, c'est moins de 22 p. Ioo de la chaleur produite qui serait alors dissipée sous forme sensible. Ces résultats sont en accord, d'une part avec les données de VAN Es et al. (1967) sur des veaux préruminants à l'en. grais (à $20^{\circ} \mathrm{C}, 4^{\circ} \mathrm{p}$. 100 de la chaleur produite sont dissipés sous forme latente et à $25^{\circ} \mathrm{C}, 60$ à 70 p. IOo,) d'autre part avec les données de McLean et CaLvert (I972) sur des bœufs de race Jersey (à $35^{\circ} \mathrm{C}, 84 \mathrm{p}$. Ioo de la chaleur perdue et $7^{8} \mathrm{p}$. Ioo de la chaleur produite sont dissipés sous forme latente).

\section{3. - Température rectale}

L'évolution de la températurc rectale pendant le stress climatique n'a pas pu être étudiée chez tous les veaux; cependant les résultats montrent que l'augmentation de la température rectale dépend de la température ambiante et surtout de l'humidité relative (fig. 2). Ce phénomène traduit la réduction de l'efficacité de la thermolyse latente lorsque la teneur en eau de l'air augmente.

L'augmentation de la température rectale varie suivant les animaux : de 0,10 à $0,32^{\circ} \mathrm{C}$ par heure pour une température de 28 à $32^{\circ} \mathrm{C}$ et une humidité relative de $80 \mathrm{p}$. 10o. Ces valeurs sont 
inférieures à celles observées par BEAKLEY et FINDLAY (1955) (0,35 ${ }^{\circ} \mathrm{C}$ par heure) chez des veaux ruminants introduits dans des chambres climatiques placées à une température de $30^{\circ} \mathrm{C}$ et une humidité relative de $80 \mathrm{p}$. roo. Chez certains animaux la température rectale atteint son maximum au bout de 6 heures, commence à baisser dès la fin du stress et retombe à sa valeur initiale au bout de 2 à 3 heures ; chez d'autres, elle augmente régulièrement et ne commence à baisser qu'une demiheure après la fin du stress.

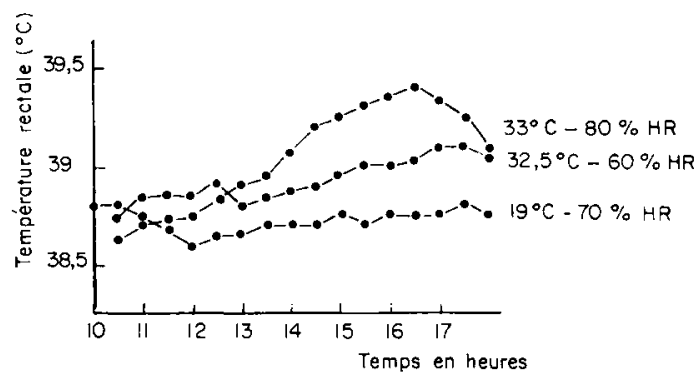

FIG. 2. - Exemple d'évolution de la température rectale au cours du stress en fonction des conditions climatiques (veau $\mathrm{n}^{\circ}$ )

\section{4. - Fréquence respiratoive}

L'influence du stress climatique sur la fréquence respiratoire a été déterminée chez 5 veaux seulement. L'élévation de la température ambiante a entraîné une augmentation de la fréquence respiratoire; de plus, pour une température donnée, l'accroissement de l'humidité relative a

\section{TABLEAU 2}

Influence de la température et de l'humidité relative ambiantes sur la fréquence respiratoire maximale atteinte et l'augmentation par degré d'élévation de température ( 2 à 5 mesures par point)

\begin{tabular}{|c|c|c|c|c|c|}
\hline \multicolumn{2}{|c|}{ Température et humidité relative } & $28 \circ \mathrm{C}-40 \%$ & \multicolumn{2}{|c|}{$28^{\circ} \mathrm{C}-71 \%$} & \multirow{2}{*}{ 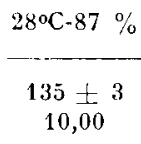 } \\
\hline $\begin{array}{c}\text { Veau } \\
n^{\circ} 480\end{array}$ & $\begin{array}{l}\text { Fréquence maximale } \ldots \\
\text { Augmentation par }{ }^{\circ} \mathrm{C} \ldots\end{array}$ & $\begin{array}{l}111 \pm 0 \\
7,33\end{array}$ & & & \\
\hline \multicolumn{2}{|c|}{ Température et humidité relative } & \multicolumn{2}{|c|}{$33^{\circ} \mathrm{C}-60 \%$} & \multicolumn{2}{|c|}{$33^{\circ} \mathrm{C}-85 \%$} \\
\hline $\begin{array}{l}\text { Veau } \\
11^{\circ} 3\end{array}$ & $\begin{array}{l}\text { Fréquence maximale ... } \\
\text { Augmentation par }{ }^{\circ} \mathrm{C} \ldots\end{array}$ & \multicolumn{2}{|c|}{$103 \pm 1$} & \multicolumn{2}{|c|}{$\begin{array}{c}139 \pm 16 \\
7,14\end{array}$} \\
\hline $\begin{array}{l}\text { Veau } \\
\text { no } 5\end{array}$ & $\begin{array}{l}\text { Fréquence maximale ... } \\
\text { Augmentation par }{ }^{\circ} \mathrm{C} \ldots\end{array}$ & \multicolumn{2}{|c|}{$\begin{array}{c}93 \pm \underset{3,57}{6} \\
\end{array}$} & \multicolumn{2}{|c|}{$\frac{141 \pm 39}{6,50}$} \\
\hline $\begin{array}{l}\text { Veau } \\
\text { no } 7\end{array}$ & $\begin{array}{l}\text { Fréquence maximale } \ldots \\
\text { Augmentation par }{ }^{\circ} \mathrm{C} . .\end{array}$ & \multicolumn{2}{|c|}{$115 \pm 4$} & \multicolumn{2}{|c|}{$\begin{array}{c}173 \pm 13 \\
8,13\end{array}$} \\
\hline $\begin{array}{l}\text { Veau } \\
\text { no } 8\end{array}$ & $\begin{array}{l}\text { Fréquence maximale ... } \\
\text { Augmentation par oc .. }\end{array}$ & \multicolumn{2}{|c|}{$\begin{array}{c}105 \pm 12 \\
3,79\end{array}$} & & \\
\hline
\end{tabular}

(La fréquence respiratoire du veau dans les conditions normales a été en moyenne de 48 respirations par minute, de 39 à 57 ). 
provoqué une augmentation importante de la fréquence respiratoire (tabl. 2), ce qui confirme les données de Riek et LEE citées par FindLAy (1950).

La fréquence respiratoire maximale est atteinte au bont de 4 à 5 heures pour une humidité relative de $60 \mathrm{p}$. Ioo et au bout de 2 à 4 heures pour une humidité relative de $90 \mathrm{p}$. Ioo. Malgré d'importantes variations individuelles, l'augmentation de la fréquence respiratoire au cours des 2 premières heures du stress est plus importante lorsque l'hygrométrie est élevée (tabl. 2 ). Les stress climatiques infligés n'ont pas conduit les veaux à la limite de leurs capacités de polypnée et n'ont pas provoqué le passage à la deuxième phase respiratoire correspondant à une respiration profonde avec risque d'alcalose.

Les observations relatives à l'augmentation de la température rectale et de la fréquence respiratoire suggèrent qu'il existe des différences importantes entre les animaux d'une même race, traduisant une différence de tolérance à la chaleur. Ces variations individuelles peuvent correspondre à des différences appréciables de comportement et de performances des veaux lors de stress répétés dans les conditions pratiques d'élevage.

\section{IV. - CONCLUSION}

Les stress climatiques relativement modérés et d'assez courte durée, se rapprochant des conditions de la pratique, n'ont pas entraîné chez les veaux préruminants à l'engrais d'augmentation excessive de la dépense énergétique, de la température rectale et de la fréquence respiratoire. Dans le cas de stress plus sévères, il serait profitable de distribuer le repas du matin plus tôt, de façon que le pic de thermogénèse se produise avant le début du stress. Ces résultats confirment le rôle très défavorable des hygrométries élevées qui s'explique par le ralentisıamınt de l'évaporation au niveau cutané et au niveau pulmonaire, la sudation étant très importante dans l'augmentation des pertes sous forme latente. Les critères les plus intéressants pour l'appréciation de la tolérance des veaux à la chaleur sont : la température rectale, l'augmentation de la fréquence respiratoire et les capacités de sudation. Enfin, l'existence des variations individuelles au sein d'une même race permet d'envisager la sélection d'animaux les plus tolérants. L'interprétation de ces variations fait appel à l'analyse des mécanismes de la thermolyse, à l'étude de la mise en œuvre progressive de ces mécanismes; elle nécessite la réalisation de mesures très diversifiées d'ordre physique, physiologique et métabolique sur les mêmes animaux et l'étude des relations entre ces paramètres.

Rę̧u pour publication en mars 1974.

\section{REMERCIEMENTS}

Les auteurs remercient vivement M. G. Theodosiadis, Station de Physiopathologie de la Nutrition et M. J. Lefaivre, Station de Recherches sur l'Élevage des Ruminants, pour les interventions chirurgicales sur les animaux.

\section{SUMMARY}

THE EFFECT ON THE THERMOREGULATION OF THE PRERUMINANT CALF OF HIGH TEMPERATURES AND HUMIDITIES FOR PERIODS OF 7 HOURS

Thirteen friesian male calves of approximately 2 to 4 months of age were submitted to the following climatic conditions : 28 or $33^{\circ} \mathrm{C}$ and 60 or $90 \mathrm{p}$. I oo relative humidity (RH) for the first 7 hours, then $19^{\circ} \mathrm{C}$ and $75 \mathrm{p}$. IOo RH for the remainder of the 24 hour day. Energy expenditure 
of the calves was determined during 39 control days and 37 experimental days, by means of two open circuit respiration chambers.

Climatic stress did not reduce feed intake of calves but induced, on an average, a 3 p. Ioo increase of energy expenditure during the time of imposition of stress and also during the day as a whole (table $\mathrm{I}$ ). The effect of stress on heat expenditure persisted for a varying time after the end of the imposition of stress ( 2 to 8 hours), according to the animals (fig. $\mathbf{I}$ ).

Total cutaneous and respiratory moisture production increased by $33 \mathrm{p}$. roo for the whole day and by roo p. Ioo for the stress period (table 1 ). Latent heat production increased with stress : 54,5 versus $42,5 \mathrm{p}$. Ioo for the whole day and 78 versus $42,5 \mathrm{p}$. Ioo for the stress period.

The respiration rate incrased with rising environmental temperature and further still with rising relative humidity (table 2 ). For a given temperature, the increase in respiration rate was highter and faster, and the increase in rectal temperature was higher, when the RH was high (fig. 2).

\section{RÉFÉRENCES BIBLIOGRAPHIQUES}

Beakley W. R., Iindlay J. C., I955. The effect of environmental temperature and humidity on the rectal temperature of calves. J. A gric. Sci, 45, 339-352.

Branca W., Hales J. R. S., x 970 . Sweating, panting and body temperatures of newborn and one-year old calves at high environmental temperatures. Br. Vet. J., 126, 45-53.

Ferguson K. A., Dowling I). F., I955. The function of cattle sweat glands. Aust. J. Agric. Res., 6, $640-644$.

Findlay J. D., I950. The effects of temperature, humidity, air movement and solar radiation on the behavjour and physiology of cattle and other farm animals. Hannah. Dairy Res. Inst. Bull., no 9.

Johnston J. E., Naelapa $H$. and Frye Jr J. B., ig63. Physiological responses of Holstein, Brown Swiss and Red Sindhi crossbred bulls exposed to high temperatures and humidities. J. Anim. Sci., 22, $432-436$

Kibler H. H., I957. Energy metabolism and cardio-respiratory activities in Shorthorn, Santa Gertrudis and Brahman heifers during growth at $50^{\circ}$ and $90^{\circ} \mathrm{F}$ temperatures. Mo. Agr. Exp. Sta. Res. Bull., $\mathrm{n}^{\circ} 643$.

KiBLER H. H., r962. Energy metabolism and related thermoregulatory reactions to thermal stress in $50^{\circ}$ and $80^{\circ}$ acclimated heifers. Mo. Agr. Exp. Sta. Res. Bull., $n^{\circ} 793$.

KLEm G. M., Robinson K. W., 1955. The heat tolerance of breeds of calves from I to i 2 months of age. Aust. J. Agric. Res., 6, 350-364.

McLean J. A., I963. Measurement of cutaneous moisture vaporization from cattle by ventilated capsules. J. Physiol., 167, 4I7-426.

McLean J. A., CalverT D. T., 1972. Influence of air humidity on the partition of heat exchanges of cattle. J. Agric. Sci., 78, 303-307.

Matthews J. S., Comfort J. E., Brody S., 1928. Growth and development. Further investigations on surface area with special reference to importance to energy metabolism. Univ. Mo. Agr. Expt. Res, Bull., $\mathrm{n}^{\circ}$ II 5 .

Van Es A. J. H., Nijkamp H. J., Van Weerden F. J., Van Hellemond K. K., I967. Energy, carbon and nitrogen balance experiments with veal calves. Proc. 4th. Symp. on Energy Metabolism. E. A. A. P. Warsaw, Poland., Pub. 12, 197-201.

VAN Es A. J. H., I968. Der Einfluss des Stallklimas auf die Produktion der landwirtschaftliche Nutztiere, insbesondere der Mastkälber, Milchkühe und Mastschweine. Aktuelle Themen der Tierernährung und Veredlungswirtschaft Lohmann, Cuxhaven, I9-26.

Vermorel M., Bouvier J. C., Bonnet Y., Fauconneau G., I973. Construction et fonctionnement de deux chambres respiratoires du type "circuit ouvert" pour jeunes bovins. Ann. Biol. anim. Bioch. Biophys., 13, 659-68г. 